\title{
IMPORTANCIA, CONTENIDO Y TRANSCENDENCIA DE LOS PAPELES DE TRABAJO DE AUDITORÍA
}

Dr. Carlos Alfonso Egúsquiza Pereda*

\begin{abstract}
RESUMEN
La importancia de los papeles de trabajo de auditoria, no solamente está dada por constituir evidencias del cumplimiento de las normas de auditoría generalmente aceptadas, de las Declaraciones de las normas de auditoria (Statements auditing standars) y las normas internacionales auditoría - NIAS, sino por el hecho de servir de prueba del control de calidad, seguido durante el proceso de auditoria, base sólida para que el auditor independiente esté en condiciones de tipificar su grado responsabilidad frente a su cliente: tipos de opinión. Por otro lado, el auditor probará con sus papeles de trabajo el cumplimiento de las técnicas y procedimientos de auditoría, por ejemplo la NIA 230 que establece normas y lineamientos respecto a la documentación sobre la auditoría de los estados financieros, el auditor deberá documentar asuntos importantes para apoyar su opinión.

Si bien es cierto, hoy en día, los papeles de trabajo tradicionales, pueden tener la tendencia de ser sustituidos o reemplazados con medios de ayuda informáticos, siempre los especialistas en auditoria preferirán utilizar aquel sistema que ofrezca mayor seguridad, por lo tanto, el sistema que esbozamos en el presente trabajo es el que tiene mayor valor agregado, ya que por ejemplo en un procesojudicial, constituirán medios más eficaces de probar la calidad de la labor efectuada por el auditor. Por ello, el presente trabajo servirá de base para que los estudiosos o profesionales puedan mejorar su desempeño como tales y sostener adecuadamente su opinióno informe.
\end{abstract}

\section{INTRODUCCIÓN}

El objeto de este trabajo es servir de pauta, de guía al estudiante o al auditor en el incremento de sus conocimientos y en el desempeño de sus funciones como profesional independiente, en la especialidad de auditoría financiera, en cuanto se refiere a los papeles de trabajo.
El presente estudio se ha creido conveniente intitularlo: Importancia, contenido y trascendencia de los papeles de trabajo de auditoria, con el fin de subrayar el valor y la importancia, que ellos merecen tener en cuenta.

Se dice que éste constituye una guía, por que no se desea imponer

* Profesor Principal de la Facultad de Ciencias Contables 
ni mucho menos intentar de dar una norma o un modelo, porque los papeles de trabajo se elaboran, se crean de acuerdo a las circunstancias y en la forma que el auditor necesite sustentar mejor sus observaciones y reparos, en un determinado trabajo.

Tampoco es intención de que el profesional, estaria impedido para basar su dictamen $u$ opinión en cuanto al cumplimiento con las NORMAS DE AUDITORIA GENERALMENTE ACEPTADAS, LOS SAS $Y$ LAS NORMAS INTERNACIONALES DE AUDITORÍA, en medios distintos a los papeles de trabajo.

Los papeles de trabajo sirven esencialmente como prueba de cumplimiento de las Normas, los SAS y NIAS de auditoria, en la ejecución del Plan de Trabajo, la revisión y apoyan el grado de responsabilidad o la tipificación de la opinión del auditor, incluyendo en el dictamen el cumplimiento de las normas, principios, los SAS y NIAS de auditoria; asi como el Reglamento sobre estados financieros auditados y las recomendaciones de la Comisión Nacional Supervisora de Empresas y Valores CONASEV.

Los papeles de trabajo son evidencias obtenidas o pruebas instrumentales elaborados durante el proceso de auditoria por el auditor independiente para mostrar los procedimientos de investigación cientifica seguidos, los muestreos llevados a cabo, las pruebas selectivas ejecutadas, los arqueos, las circularizaciones, las pruebas alternativas y pruebas especiales aplicadas de acuerdo a las circunstancias; la información que obtuvo, las limitaciones a que estuvo expuesto su trabajo; por último, las conclusiones a los cuales llegó, con relación al examen practicado. Incluirán por lo tanto, pruebas del cumplimiento a cerca de la evaluación de los sistemas administrativo, operativo, contable, de costos y computarizado de datos (cuestionarios y/o evaluación de sistemas*), el programa de trabajo o procedimientos mínimos que cumplió el auditor, balance de comprobación, anexos o análisis de cuentas, proporcionadas por el cliente, balance de situación financiera y estado de ganancias y pérdidas, estado de cambios en la situación financiera o estados de flujo efectivo, estados de cambios en el patrimonio neto, asientos de ajustes y reclasificación, circularización y confirmación de los saldos, resumen de las lecturas practicadas a los libros de actas de Directorio y Junta General de Accionistas, carta de confirmación de Directorio o Gerencia, etc. De la misma manera el auditor debe recabar información suficiente en sus papeles de trabajo a cerca de la evaluación que ha practicado, relativo a la habilidad de una entidad para continuar como negocio en marcha, que establece el SAS 59.

Para cumplir con el propósito de este estudio se ha creído conveniente dividir el tema en cuestión en cuatro partes:

1. Objetivos en la preparación de los papeles de trabajo.

2. Tipos de papeles de trabajo.

3. Archivo de los papeles de trabajo. Clases

4. Conservación de los papeles y registros de auditoria.- Propiedad

5. Conclusiones y recomendaciones. 


\section{OBJETIVOS DE LA PREPA- RACIÓN DE LOS PAPELES DE TRABAJO}

Los objetivos al preparar los papeles de trabajo de auditoria son para que sirvan de evidencia competente, suficiente e irrefutable, prueba instrumental de base para la tipificación de la opinión o sea cimientos sólidos para la toma de decisiones en la auditoria.

Los datos y observaciones consignados en los papeles de trabajo apoyan los principios, normas y procedimientos de auditoría, seguidos y apegados durante el examen. Porque, ellos apoyan el ámbito y la opinión del auditor independiente en su informe. En muchos casos al principio de la preparación de los papeles de trabajo puede anteceder al estudio de los sistemas administrativo, contable, operativo, de costos y computarizado de datos (antes evaluación el control interno administrativocontable) y el examen de la evidencia puede conducir a la programación de los mismos.

Los objetivos de los papeles de trabajo, son indudablemente, demostrar en forma clara el procedimiento seguido en la revisión, el alcance del examen, y los respectivos comentarios, conclusiones y recomendaciones a las que se ha llegado; que a su vez generan en última instancia en la preparación del informe o dictamen.

\section{a) Propósitos:}

En cuanto se refiere a los propósitos de los papeles de trabajo podemos mencionar los siguientes:

1. Tienen por objetivo principal de ayudar al auditor en la tipificación de la opinión del auditor o sea que tipo de dictamen le va a entregar a la empresa o cliente.

2. Sirven de respaldo al auditor en caso de algún proceso judicial, el auditor puede sustentar su defensa en asuntos que implique negligencia o fraude, mediante la prueba de papeles de trabajo.

3. Sirven de nexo entre los registros y datos del cliente y el informe del auditor.

4. Otro de los propósitos es que ellos indican el grado de confiabilidad de los sistemas puesta en práctica por la empresa durante la gestión de la empresa y la elaboración de la contabilidad y de los estados financieros.

5. Sirven de fuentes o datos en las discusiones personales en asuntos financieros, económicos o comerciales con los funcionarios de la compañia.

6. Apoyan al auditor en la solución de los problemas que se pueden presentar en las diversas entidades estatales o privadas en la preparación de los estados financieros o consulta dé algún dispositivo legal;

\section{b) Contenido:}

En cuanto al contenido de los mismos y dichos de manera muy sucinta, compilados en una revisión independiente, contendrán o incluirán las siguientes cédulas, que en el punto 2.0 Tipos de papeles de trabajo, se estudian los más importantes:

1. Una copia a lápiz del dictamen e informe largo (diagnóstico de los sistemas imperantes, antes de la carta de control interno de gerencia) 
2. Balance de situación financiera comparativo antes y después de la auditoría

3. Estado de ganancias y pérdidas comparativo antes y después de la auditoria

4. El balance de comprobación y los anexos proporcionados por el cliente.

5. Relación de los asientos de ajuste y reclasificación de auditoria.

6. Relaciôn de análisis de activos, pasivos, capital y de estados de ingresos $\mathrm{y}$ gastos.

7. Circularización y confirmación de bancos, cobrabilidades y de otras cuentas de activos y pasivos.

8. Copia de los estatutos, y modificatoria de los mismos.

9. Organigramas y manuales de funciones y responsabilidades.

10. Copia de contratos importantes.

11. Resumen de las actas de la Junta General de Accionistas y acuerdos del Directorio que tengan relación con las cuestiones financieras y económicas.

12. Reconciliaciones bancarias.

13. Copias de las Declaraciones Juradas de Impuesto a la renta de los dos últimos ejercicios.

14. Procedimientos y programas de auditoría.

15. Cuestionarios y flujogramas de la evaluación de los sistemas.

16. Carta confirmatoria de Gerencia o Directorio, sobre:

a) Inventarios

b) Activos en general

c) Pasivos, etc.
17. Cuadros de cálculos de provisión para compensación por tiempo de servicios para obreros y empleados, etc.

18. Pruebas practicadas con respecto a los eventos posteriores y las contingencias, etc.

Por último, para terminar este punto, debemos referirnos a la propiedad de los papeles de trabajo, que todos los papeles acumulados durante el examen, es propiedad del auditor. Sin embargo, el auditor puede proporcionar una copia al cliente si es que considera pertinente; sin que éste exija que se le proporcione todo, dejando al auditor sin ningún ejemplar.

En el caso de un examen de estados financieros de entidades Estatales, está impedido de mostrar o exhibir los papeles de trabajo, por lo tanto es propiedad única y exclusivamente del auditor; salvo que ordene la exhibición o entrega de los papeles el Juzgado.

\section{c) Principios en la preparación de los papeles de trabajo}

Se denomina principios en la preparación de los papeles de trabajo al conjunto de experiencias, iniciativas $y$ habilidades para formular los papeles de manera adecuada y suficiente; o sea cuando se ha tenido en cuenta, durante la formulación, los siguientes conceptos genéricos: $\mathrm{Si}$ el auditor se ha apegado a los principios normas y técnicas de auditoria generalmente aceptados; si los papeles han pasado por la supervisión, revisión de otro auditor o supervisor con suficiente experiencia; y luego, si este último puede formular el informe de auditoría. Se habrá cumplido con 
todas las pruebas de excelente calidad en la preparación.

Estos principios que rigen en la compilación de los papeles de trabajo se resumen de la siguiente manera:

1. Todos los datos incluibles o pertinentes deben ser considerados en su totalidad.

2. Los datos no incluibles o no significativos no se deben considerar.

3. Se incluirán todos los datos que puedan apoyar algún concepto, afirmación $\mathrm{u}$ observación.

4. Se efectuarán las anotaciones que puedan servir en lo futuro.

5. Se incluirán datos relacionados a la calidad de los registros realizadas en los libros contables, así como de los archivos del cliente.

6. Los papeles de trabajo deben indicar, una enunciación, o concepto del trabajo practicado en conexión a la preparación de los mismos.

En el transcurso del examen todos los papeles de trabajo deberán ser controlados, protegidos y luego conservados, adecuada y suficientemente. Debe señalarse e indicarse al personal de auditoría la responsabilidad a compartirse. Al terminar cada papel deberán firmarse por las personas que hicieron la tarea; además deberán estar revisados y firmados por el supervisor o socio.

Es necesario también, hablar del empleo de un método adecuado para el archivo y presentación correcta. Es indispensable todo esfuerzo empleado con la finalidad de uniformar y mejorar los tipos de papeles de trabajo y las marcas y claves utilizadas.
Además de los principios que acabamos de mencionar, se debe tener en cuenta las normas de auditoría generalmente aceptados en la formulación y preparación de estos:

1. Normas generales o personales.

2. Normas relativas a la ejecución del trabajo en el campo.

3. Normas relativas al informe o dictamen.

Estas tres normas están directamente $e$ indirectamente relacionadas con los papeles de trabajo.

Las normas de ejecución del trabajo, que forma parte de las normas de auditoría generalmente aceptados, requiere de una adecuada planeación, supervisión y control; un estudio o diagnóstico de los sistemas y una obtención de evidencias suficientes y competentes; insistimos en esto, porque los papeles de trabajo para el auditor son consideradas como médula en sus diversas funciones $\mathrm{y}$ actuaciones.

Los estados financieros están normados por el balance de situación ajustado, que levanta el auditor sobre la base del balance de comprobación y está formado por las cuentas del activo, pasivo y capital ordenados de acuerdo al grado de liquidez o el grado de realización o extinción de la deuda. En un balance comparativo con la del año anterior, en la que se puede mostrar las variaciones -incrementos o disminuciones-sufridos en los activos y pasivos y sobre todo el incremento del capital social y las utilidades. Generalmente, se hace la presentación en miles de soles. Algunos puntos en las que el auditor ha necesitado salvar su opinión o ha querido hacer 
algunas observaciones sobre rubros del activo o pasivo, pueden estar acompañados de las notas correspondientes. Comparado el balance presentado por el cliente y el formulado por el auditor, generalmente va a tener una diferencia bastante saltante, no solo por los asientos de ajuste, si no también debido a los asientos de reclasificación, que se hicieron para una razonable o mejor presentación.

\section{d) Dictamen}

Son las hojas que contienen la redacción a lápiz del dictamen o informe; es la expresión del auditor sobre los estados financieros examinados a una fecha determinada. Que manifiesta o divulga el alcance de la revisión, los procedimientos seguidos y la opinión razonable o no de los estados financieros y la veracidad de sus resultados operaciones económicas. El auditor para poder tipificar su opinión debe utilizar ciertas reflexiones técnicas y científicas, para ello, por ejemplo puede utilizar el enfoque que estoy consignado mediante la estructura y contenido de los variables o parámetros que el auditor debe estudiar, teniendo en cuenta los papeles de trabajo, esta cédula se archivará en el archivo general corriente-general current file.

\section{e) Programa de Auditoría}

Es la cédula que contiene el plan total de trabajo, guías de acción por cada una de las cuentas o rubros examinados, en aplicación de las técnicas y procedimientos de auditoría seleccionadas en armonía a las normas de auditoría, los SAS y las NIAS, los que se ilustran en cada una de las cêdulas básica se incluyen en el tercer tipo o grupo de archivos.

\section{TIPOS DE PAPELES DE TRABAJO}

Las hojas de trabajo tomados como elementos de juicio de auditoría, resulta evidente que ellos han de tener una gran variedad de formas y de disposiciones, de acuerdo al juicio del auditor. Desde los habituales hojas de papel en columnado utilizando en la contabilidad para asientos de diario, balance de comprobación, de cuadros de ganancias y pérdidas; hasta simples hojas de papel de notas tomados de los contratos, reuniones que pueden ser papeles especiales o simples hojas de 7 columnas cuadriculas o rayadas.

Aún, cuando los papeles de trabajo son tan diversas, hay ciertos que son bastante comunes. Algunas firmas profesionales han creado estilos tradicionales de hojas de trabajo que han encontrado altamente satisfactorias y recomiendan emplear a su personal. Una de las características o cualidades del auditor debe ser la capacidad para idear y crear nuevos tipos de papeles de trabajo, adecuado a las necesidades de los problemas de verificación o recolección de información.

El mejor tipo, será indudable. aquel que combina la necesidad de acopio de información, con el de verificación de información reunida. En todo tratado sobre auditoria y en las convenciones internacionales de la profesión, han recomendado siempre, la sugerencia sobre la manera de encarnar el problema y no de modelos que puedan adoptarse 
en todo los casos, se debe estar atento al cambio, a la modificación, cuando se produce alguna situación especial, en situación más complejas, se debe levantar hojas de acuerdo a las circunstancias y las condiciones del Convenio suscrito con el cliente.

\section{a) Estados Financieros}

La ejecución que el auditor debe seguir con el objeto de cumplir con el convenio, en su intento o afán de reunir suficiente información como para elaborar un informe razonable.

El programa de trabajo es conseguido por el auditor jefe o encargado como un elemento de juicio y raciocinio, antes, durante $y$ después de la revisión. Generalmente está supeditada a las siguientes condiciones:

1. Opinión a emitir,

2. Particularidades de la empresa en estudio,

3. Grado de confianza del control interno.

4. Planificación de los diversos procedimientos a seguirse, en forma tal de que los colaboradores puedan seguir durante el examen, mediante las instrucciones escritas que se incluyen.

Como papel de trabajo es importante, porque presenta: Primero el diagrama seguido por el equipo de auditoría, el alcance de la labor realizada; segundo, porque sirve como constancia de la realización efectiva de las etapas previstas o programadas. Inclusive, sirve para evaluar el avance del trabajo y la calidad de la misma. La extensión o amplitud depende, indudablemente del control interno imperante en la empresa y el convenio suscrito.

\section{b) Balance de Comprobación}

Denominada comúnmente, "balance de comprobación de trabajo", es el meollo de las hojas de trabajo de la auditoria, todos los demás papeles $\mathrm{u}$ hojas convergen y divergen de él, inclusive los asientos de ajustes y reclasificación se vuelcan al balance de comprobación. En mérito a esta hoja se prepara el balance general o estado financiero y económico con el objeto del informe final.

Se estila diversas formas según los auditores en una $u$ otra forma $o$ método tiene sus ventajas y desventajas.

- Constituye la transcripción de la cuenta del libro mayor, con saldos pertinentes o saldos nulos, por lo que constituye la mejor fuente de información;

- Comprende las siguientes columnas:
a) Número de la hoja de trabajo,
b) Saldo de las cuentas según el mayor del ejercicio ante- rior al examinado;
c) Movimiento y saldo de las cuentas, según el mayor del ejercicio en examen;
d) Saldo según balance del Cliente,
e) Ajustes y reclasificaciones de auditoria, cargos y abonos,
f) Saldos según Auditoría.

\section{c) Ajustes y reclasificaciones de Auditoria}

Los estados financieros sometidos a examen indudablemente, están 
sometidos al descubrimiento de errores o irregularidades en la información financiera, que requiera de la corrección mediante asientos de ajustes y reclasificación. Esta tarea la realiza el auditor mediante la preparación de una lista de asientos que recomendará a su cliente para que sean incluidos en libros contables; esto se consigue mediante la preparación de la hoja de trabajo única y exclusivamente para este fin. Mediante estos asientos se va a poder tener las cifras de comprobación llamados SALDOS SEGÚN AUDITORÍA.

El control interno debidamente evaluado constituye una fuente básica del auditor, que sirve para medir la extensión y alcance del examen, inclusive para fijar los honorarios profesionales, o sea en otros términos la aplicación de una sana política de control interno, por una gerencia cauta, conducirá dentro de la aceptación de la profesión al menor despliegue de conocimientos y elementos humanos durante el examen de un ejercicio determinado.

\section{d) Otras hojas de Trabajo}

Entre otras hojas de trabajo tenemos los siguientes:

- Análisis de cuentas,

- Conciliaciones Bancarias,

- Extractos y notas de actas de Asamblea de Directorio y Junta General de Accionistas,

- Catálogo de cuentas y política contable,

- Copia de contratos importantes,

- Circularización de cuentas por cobrar y pagar.
- Carta confirmatoria de gerencia sobre activos y pasivos, etc.

\section{ARCHIVO DE LOS PAPELES DE TRABAJO. ClASES}

Los Archivos en la auditoría se dividen en Archivos Permanentes (permanent file) y Archivo Corriente (current file) y Archivo General Corriente (General current file). A continuación vamos a dedicar al estudio cada uno de estos archivos por separado, el personal de auditoría debe estar familiarizado con el manejo y cuidado de estos archivos, por concentrar datos, documentos valiosos y que sirven de amparo y respaldo al profesional.

\section{A) Archivo Permanente}

El objeto de este archivo es de singular significación. Incluye datos y documentos de interés no sólo ejercicio en revisión sino a futuros ejercicios, historia de la empresa los propósitos con los siguientes:

1. Conocimiento por parte de personas ajenas en asuntos del cliente, pueden rápidamente conocer el desarrollo normal de operaciones financieras y económicas.

2. Proporciona el resumen de partidas, de manera inmediata, evitando la revisión de algunos rubros;

3. Sirve para evitar de preparar nuevas cédulas sobre rubros de poco o ningún movimiento;

4. Agenda para separar y organizar datos especializados para auditorias subsecuentes. 
En el archivo permanente se encuentran anotaciones del auditor que van a servir para la próxima revisión. En caso de funsión a otra empresa o reorganización, el archivo permanente juega un papel importante, porque proporciona la historia de la entidad y porque da una explicación sobre activos $y$ utilidades.

El contenido del archivo permanente puede ser el siguiente:

\section{a) Historia de las Informaciones y datos generales:}

- Historia de la empresa, expansión, financiamiento, cambio de giro, organización, etc.

- Organigrama estructural y funcional de la empresa.

- Escritura de constitución, modificatoria de los estatutos

- Copia de contratos importantes, proyecto de distribución de excedentes convenios sindicales, etc.

- Cuestionario de control interno y auditoria interna.

- Política contable.

- Copia del registro de Acciones.

- Plan o catálogo de cuentas.

- Procedimientos de inventarios.

- Copia de Declaraciones juradas sobre impuestos.

- Circularización de cuentas por pagar y cobrar,

- Extractos de Actas de Junta General de Accionistas y acuerdos importantes del Directorio.

- Contrato de Alquileres.

- Principales formatos y papeles utilizados por el cliente, etc. b) Resumen de análisis de las cuentas más importantes y de tipo permanente:

\begin{tabular}{ll}
\multicolumn{1}{c}{ ACTIVO } & \multicolumn{1}{c}{ PASIVO } \\
- Inversiones a largo Plazo & - Pasivo a Largo Plazo \\
- Terrenos & - Reserva del Capital \\
- Edificios & - Capital \\
- Maquinarias & - Superávit pagado \\
- Muebles y enseres & - Superávit Ganado \\
- Provisión para Depreciaciones & - Ventas plazos \\
- Intangibles & \\
- Cargas diferidas &
\end{tabular}

\section{B) Archivo Corriente}

$\mathrm{El}$ archivo corriente viene a hacer previo al archivo permanente, en ella se incluye todos los datos y documentos relacionados al ejercicio fiscal o al ejercicio en examen y a medida que va pasando el tiempo de un ejercicio a otro va trasladando el ARCHIVO PERMANENTE.

El contenido minimo de este archivo es el siguiente:

- Cita de asuntos pendiente que resolver,

- Cuestionario de control interno,

- Correspondencias:

- Modificaciones de la Escritura de Constitución, Estatutos.

- Extractos de Actas.

- Papeles de trabajo de la Balanza de Comprobación,

- Dictamen o informe de Auditoría,

- Programas de Auditoria,

- Cédulas numéricas y analiticas,

- Ajustes y reclasificaciones de auditoría, en algunos casos asientos de cierre y apertura.

- Reporte de tiempo y gastos. 


\section{CONSERVACIÓN DE LOS PAPELES Y REGISTROS DE AUDITORIA}

En cuanto a la conservación de los papeles y registros de auditoria deben conservarse no menos por cinco años, guardándose con celo cuidado, pero por razones de ética, unas veces por razones de cumplimiento de trabajo posteriores o futuros con el cliente se debe seguir la siguiente conservación, de acuerdo a la importancia y trascendencia que pueda tener cada una de las cédulas de auditoria.

\section{a) Conservación Permanente}

Dentro de los diversos papeles y cédulas que se conserva de manera permanente son los siguientes:

- Una copia mecanografiada de cada informe de auditoria,

- Papeles necesarios para dar validez a los estados financieros del cliente,

- La discriminación de las cuentas de capital en su totalidad,

- Declaraciones Juradas sobre impuestos en general,

- Correspondencias importantes de naturaleza permanente,

- Contratos en vigencia,

- Manual de Registros de Contabilidad, plan de cuentas, escritura de constitución, estatutos y modificaciones, Muestreo de certificados de acciones y bonos activos.

b) Conservación durante diez años

- Extractos de Actas.

- Análisis detallado de los motivos fijo

- Análisis pormenorizado de las cuentas de ingresos y gastos

- Contratos vencidos,

- Informes de auditoria Interna, c) Conservación durante 5 años

- Conciliaciones bancarias y circularización de los saldos,

- Balance de comprobación de las cuentas y documentos por cobrar y pagar,

- Respuesta a las confirmaciones de las cuentas y documentos por cobrar y pagar.

- Análisis de los débitos y créditos diferidos

\section{d) Conservación durante 3 años}

- Correspondencia rutinaria,

- Copias adicionales de informes mecanografiados

- Borradores de los informes

\section{CONCLUSIONES}

1. Los papeles de trabajo constituyen la base fundamental para la toma de las decisiones en la auditoria.

2. Los comentarios, observaciones y conclusiones que figuran en los papeles de trabajo apoyan los principios, normas y procedimientos de auditoria generalmente aceptados. Amparan el alcance de la revisión y la opinión del auditor.

3. Los propósitos de los papeles de servir de fuente entre los documentos y registros del cliente y el informe del auditor; mediante ellos se puede medir el grado de confianza del control interno y la eficacia de la política administrativa de la gerencia. Sirve de apoyo y respaldo al auditor en cualquier actuación dentro de la empresa o fuera de ella y aún más en caso de acción judicial, por razones de negligencia o fraude.

4. El contenido varía de acuerdo al tipo de auditoria que se practique y a la envergadura de la empresa, 
compilados y archivados cuidadosamente no podrán dejar de incluir un mínimo de cédulas suficientes como para levantar un informe.

5. Los Principios que rigen en la compilación son de imprescindibles de aplicación y estos son: claridad, no debe haber datos dudosos o que encierren falsedad; contendrá datos que apoyen la propiedad de los registros del cliente y tendrá una enumeración del procedimiento seguido en la recolección de informaciones $\mathrm{y}$ datos.

6. Los tipos de los papeles de trabajo enunciados constituyen lo más saltante, además hay otros tipos que surgen de acuerdo al criterio y la clase del trabajo que realiza el auditor. Hay papeles de generación interna y de generación externa, o sea que nacen en la empresa y fuera de ella.

7. Los archivos constituyen historia detallada del cliente, cuyo manejo necesita la especialización y familiarización del personal de auditoría por contener datos y documentos que avalan al prestigio y la responsabilidad del auditor independiente.

8. Los Archivos de los papeles usualmente se clasifican en PERMANENTES Y CORRIENTES. Establecidos con el objeto de facilitar, ordenar y actualizar la cédulas, al pasar de un ejercicio a otro automáticamente se convierten en permanentes.

9. Por razones de ética, por razones de cumplimientos de trabajos posteriores con clientes y de acuerdo a la práctica, hay documentos, datos y registros que se conservan unas permanentes $\mathrm{y}$ otras por espacio de diez, cinco y tres años.

\section{RECOMENDACIONES}

1. En las Universidades y en los colegios profesionales deben establecerse laboratorios talleres de práctica única y exclusivamente para discutir y realizar prácticas sobre los papeles de trabajo.

2. El personal de auditoria debe perfeccionarse mediante cursos, talleres, seminarios de manera continua, para mejorar la calidad y el contenido de los papeles.

3. A los auditores y ayudantes se deben proporcionar separatas e instrucciones escritas con el objeto de mejorar y ahorrar tiempo y entre estas serian las siguientes:

- Identifique claramente cada hoja,

- Utilice hoja separada para cada asunto y escriba en una sola cara,

- Identifique al supervisor,

- Use referencias claves,

- Incluya información completa y específica.

- Anote la naturaleza, extensión y alcance de las pruebas selectivas aplicadas,

- Mantenga una hoja separada para asuntos que necesitan recordar o aclarar.

- Incluya comentarios, observaciones y conclusiones para cada rubro examinado, revisado, etc.

4. El auditor independiente debe optar procedimientos especiales para proteger sus papeles de trabajo.

5. En la medida de las posibilidades las compañias auditoras deben tratar de estandarizar sus papeles de trabajo. 
6. Los auditores por ningún motivo deben dejar los papeles de trabajo en la oficina de clientes.

\section{BIBLIOGRAFIA}

1. Dr. Larry P. Bailey, Phd., Dr. Oscar J. Holzmann, PhD., Traductor : 1998-1999 Guía de Auditoria, Editorial Harcourt Brace. España.

2. Instituto Mexicano de Contadores Públicos A.C. Federación de Colegios Profesionales, 1998, Normas Internacionales de Auditoria.

3. Instituto Mexicano de Contadores Públicos A.C. Comisión de Normas y Procedimientos de Auditoria, 1998, Normas y Procedimientos de Auditoría. Tomo 1 y 2.

4. XXIV Conferencia Interamericana de Contabilidad. Noviembre del 2001, Punta de Este Uruguay. Trabajos Técnicos Interamericanos.

5. Instituto Mexicano de Contadores Públicos A.C. Federación de Colegios Profesionistas, 1997, Declaraciones sobre Normas de Auditoria emitidas por el Consejo de Normas de Auditoría. Tomos del 1 al 7.

6. XXIII Conferencia Interamericana de Contabilidad. Agosto de 1999, San Juan de Puerto Rico. Trabajos Técnicos Interamericanos.

7. J.A. Cashin, P.D. Neuwirth y J.F. Levy.- Manual de Auditoria, tomos: I, II y II BMC Biblioteca Master Centrum, Editorial Océano, España.
8. Principios de Auditoria.- Walter B. Meigs

9. Programas de auditoria: Sánchez Alarcón.- ECASA - México 1991

10. Elementos de auditoria. Mendivil Escalante Víctor: México 1992

11. Normas de Auditoría Gubernamental-Oficina General de los Estados Unidos (U. S. General Accounting Office), junio de 1994, Traducción de la Contraloría General de la República del Perú - GAO.

12. Código de Ética profesional del Colegio de Contadores Públicos de Lima Reglamento para la preparación de la informaciôn financiera de la Comisión Nacional Supervisora de Empresas y Valores - CONASEV No 103-99$\mathrm{EF} / 94.10$

13. Diccionario para Contadores, Eric L. Kohler, Unión Tipográfica Editorial Hispano Americana UTHEA - México 1980.

14. Guía de Auditoría, Normas de Auditoría (SAS), (Miller) DR. Larry P. Bailey, Dr. Oscar J. Holzmann, Editorial Harcourt Brace, España 1998.

15. Compendio de Legislación del Sistema Nacional de Control, Contraloría General de la República, Perú, Gaceta Jurídica, 1996.

16. Normas de Auditoría Gubernamental-NAGU-Government Auditing Standards. Contraloria General de la República del Perú, Setiembre de 1995. 